\title{
THE ORIGINS OF IRREVERSIBILITY
}

\author{
E. TIEZZI \& N. MARCHETTINI \\ Department of Chemical and Biosystems Sciences, University of Siena, Italy.
}

\section{ABSTRACT}

This article is a theoretical contribution dedicated to Boltzmann's theorem revisited in light of the studies of Mario Ageno, late professor of Physics at the University of Rome (La Sapienza), who showed an interesting new point of view on probability and uncertainty in his book The Origins of Irreversibility. The discussion takes into account the equation of Ilya Prigogine, Nobel Prize winner in Chemistry, for the calculation of entropy production per unit time. The main point is that the authors claim that there are three different conditions: (a) the tendency towards disorder (Boltzmann) as in the case of gas; (b) the Ageno contribution related to the collisions of molecules against the walls: in this case Ageno claims that the origin of irreversibility derives from the tendency towards a macrostate richer in microstates; (c) the presence in the liquids, living systems and water of possible dissipative structures: in this case self-organization is the most probable state (Prigogine's order out of chaos) and the tendency is towards an ordered state.

Keywords: Boltzmann's theorem, entropy, irreversibility, order out of chaos

\section{INTRODUCTION}

Irreversibility is a milestone concept both in thermodynamics and evolution and, now, in ecology, due to the determinant role of global warming.

In thermodynamics, irreversibility is related to the concept of entropy and to the natural spontaneous trend towards disorder and not vice versa. According to the second law of thermodynamics, a machine cannot transfer heat from a cold body to a hot one without performing work. Whenever work is produced from heat, heat also passes from a hot body to a colder one. Our everyday experience with devices (from motors to electric razors) shows us that work is inevitably accompanied by heating which was not the intention of the machine. There is a tendency in the universe towards the 'heat form' of energy. Heat is a 'degraded' form of energy because it cannot be totally converted back into work. Only some of the heat can be transformed into work; we cannot freely recover heat from a cold body. For example, the ocean is an immense store of heat; it contains an enormous quantity of energy, but we cannot use it freely. Although it contains much more heat than the human body, we cannot warm our hands by it because the ocean is a colder source than our hands and heat cannot pass spontaneously from a cold to a warmer body.

At this point, we could open a parenthesis on what we might call 'Maxwell's demon revisited'. In 1871, J.C. Maxwell proposed a paradox which embarrassed physicists for a long time. He imagined a system with gas in two containers A and B at the same temperature, separated by a wall. There was a small aperture in the wall guarded by a demon that separated fast moving molecules from slow moving ones (i.e. hot molecules from cold ones as temperature is a measure of the movement of molecules), putting the first into A and the second into B. In the end, there would be a temperature difference in contradiction to the second law of thermodynamics. N. Georgescu-Roegen (a scientist of Rumanian origin who taught economics in the US and was known for his application of the principles of thermodynamics to economics [1]) observes that we now consider Maxwell's demon to be exorcised; like any other living creature, the demon must use more energy than it creates by separating hot and cold molecules. He adds that many theories on the unlimited renewability of resources imply a demon having miraculous faculties behind the scenes.

(C) 2007 WIT Press, www.witpress.com

ISSN: 1743-5242 (paper format), ISSN: 1743-5250 (online), http://journals.witpress.com DOI: $10.2495 / E C O-V 2-N 3-165-169$ 
The role of entropy in biological evolution is obviously fundamental. Entropy brings into biology the concept of a direction in time, namely that time prefers to flow from past to future, from lesser to greater entropy. Time and complexity become the protagonists of our era, imposing different frameworks for natural, economic and social phenomena. Nature shows us that her processes are irreversible. 'Irreversibility and uncertainty are the rule', says Prigogine. On this point Prigogine writes in La Nouvelle Alliance [2]:

Life, regarded as the result of [improbable] initial conditions, is in this sense compatible with the laws of physics (the initial conditions are arbitrary), but does not follow from the laws of physics (which do not set the initial conditions). This is the view of Monod. Furthermore, life, from this point of view, looks like a continual struggle by an army of Maxwell devils against the laws of physics, to maintain the highly improbable conditions which allow it to exist. Our point of view is completely different in that vital processes, far from being outside nature, follow the laws of physics, though in specific non-linear interactions and in conditions far from equilibrium. These aspects can in fact provide the flow of energy and material necessary to build and maintain functional and structural order.

Ilya Prigogine is the main father of the idea of irreversibility. His autobiography recounts his first studies on far-from-equilibrium phenomena:

It is difficult today to give an account of the hostility that such an approach was to meet. For example, I remember that towards the end of 1946, at the Brussels IUPAP meeting, after a presentation of the thermodynamics of irreversible processes, a specialist of great repute said to me, in substance: 'I am surprised that you give more attention to irreversible phenomena, which are essentially transitory, than to the final result of their evolution, equilibrium.'

He adds:

As we started from specific problems, such as the thermodynamic signification of non-equilibrium stationary states, or of transport phenomena in dense systems, we were faced, almost against our will, with problems of great generality and complexity, which call for reconsideration of the relation of physico-chemical structures to biological ones, while they express the limits of Hamiltonian description in physics. Indeed, all these problems have a common element: time.

The intrinsic irreversibility of time has erupted in the basic equations of chemistry and physics. More than a hundred years have passed since Max Planck, completely alone in the Vienna Meeting, underlined the singularity of the heat form of energy; it is more than a hundred years since Sadi Carnot, at the age of 28 years, opened the way to the concept of entropy. The dominant socioeconomic theories continue to ignore the existence of entropy.

\section{DISCUSSION: THE AGENO CONTRIBUTION}

Mario Ageno, late professor of Physics at the University of Rome (La Sapienza), showed an interesting new point of view on probability and uncertainty in his book The Origins of Irreversibility [3].

Ageno underlines that at each collision of a molecule against the walls, there is a non-zero probability of transition to any of a continuum of microstates, and due to the unstable character of the trajectories in phase space, all microstates possible a priori for the gas therefore become accessible. This is the only reason why most times the gas is observed and for most of the time it is observed, it is found to be in that phenomenological (macroscopic) state that covers a much greater number of microstates than all other states of the kind, namely the state of thermodynamic equilibrium. If the 
gas is going through an obligatory succession of microstates, $b_{i} \rightarrow b_{i+1} \rightarrow b_{i+2} \rightarrow \ldots$, the transition from one microstate to the next is caused by an elastic collision between two molecules. When the gas is in microstate $b_{i+k}$, there comes a moment when one of its molecules collides with a wall of the container. The inelastic collision causes a transition to another obligatory succession of microstates, $c_{1} \rightarrow c_{2} \rightarrow \ldots$, completely independent of the former succession and microstate $c_{1}$ will be chosen randomly on the basis of the laws of probability from a continuous range of possible microstates. The most probable choice is a microstate belonging to the macrostate richest in microstates in the range. Of course, this analysis is completely in line with classical statistical thermodynamics and with the classical related concept of probability.

Ageno adds,

imagine inverting the direction of all molecular velocities while the gas goes through the obligatory succession of microstates $b$, the succession will run in the opposite direction $b_{i} \rightarrow b_{i-1} \rightarrow$ $b_{i-2} \rightarrow \ldots$. However, there comes a time when the transition from a certain microstate $b_{i-b}$ to the next is caused by collision of the molecule against the wall. Again, this causes a transition to another obligatory succession of microstates, $a_{n} \rightarrow a_{n-1} \rightarrow a_{n-2} \rightarrow \ldots$, quite independent of succession $b$, and now microstate $a_{n}$ is chosen at random from the continuous range of alternative possibilities.

Once again, the most probable choice falls on a microstate belonging to the macrostate richest in microstates in that range. We see that in whatever direction the gas goes through part of the obligatory succession of microstates, collision of one of its molecules against the walls will always tend to make it go from the macrostate in which it was before the collision to another macrostate richer in microstates. This transition is the most probable event when the gas leaves the obligatory succession in which it was. By frequent collisions of molecules against the walls, most of the times we observe the gas and for most of the time we observe it, the gas will therefore be found in the macrostate richest in microstates, namely the state of thermodynamic equilibrium.

According to the paradigm of gases, we see that the laws governing elementary processes can identify a linear order of events occurring at that level but cannot attribute a direction to the linear order. In the case of the deterministic model gas of Boltzmann, the laws of mechanics enable us to establish that the three microstates $a, b$ and $c$ belong to the same obligatory succession of the gas. For example, microstate $b$, is between states $a$ and $c$, but the laws cannot tell us which is first and which is later, whether $a$ precedes $c$ in time or whether $c$ precedes $a$ in time. According to the laws of mechanics, there is perfect symmetry between the two cases.

Now, the event that separates the time spent in a microstate from that spent in the adjacent microstate is in any case a molecular collision. There are always two collisions at the two limits of the time the gas spends in a given microstate. According to Ageno, "if one is a collision with the walls of the container, there is a transition from one obligatory succession of microstates to another quite independent succession immediately adjacent to it in time on both sides.'

In the first collisions with the walls in a given order, we may find that the previous macrostate was particularly poor in microstates and becomes richer as a result of the collision. Collision after collision, the gas on average achieves richer and richer macrostates, each time choosing between the most probable available. This means that the gas evolves towards a state of equilibrium: the order arbitrarily chosen in the chain of microstates is the one that will most probably occur physically and it is indicated by the arrow of time.

If, on the other hand, in the order we arbitrarily chose, we find that the gas on average goes to increasingly improbable macrostates, increasingly poor in microstates, from collision to collision with the walls. Ageno underlines [3], 'Clearly that order has no appreciable probability of occurring 
physically in the segment of the chain of microstates we considered: the arrow of time points in the other direction.'

It is therefore the continuous character of the range of choices open to the gas at every collision that gives rise to asymmetry between the two directions in which the chain of microstates is followed: a probability, proportional to the number of microstates in each available macrostate, is attributed to the two directions each time. However, the continuity of the range of choices springs in turn from the probabilistic character of the phenomena of absorption and emission of electromagnetic radiation by the gas. The irreversibility of the macroscopic processes therefore originates in the uncertainty between energy and time. This analysis is completely opposite to the idea of order out of chaos by Prigogine.

This new Ageno's interpretative framework of the second law of thermodynamics and the origins of irreversibility of macroscopic processes, with recognition of the stochastic nature of the inevitable residual 'perturbations', offers a reasoned foundation for the use of probabilistic methods in statistical mechanics and in classical physics in general. However, this aseptic concept of probability does not consider the capacity of living systems to self-organize. The role of collisions against the walls is, of course, important.

Ageno [3] is wrong when he writes: 'Clearly that order has no appreciable probability of occurring physically in the segment of the chain of microstates we considered: the arrow of time points in the other direction.' It is the temporal sequence of the events, which determines the arrow of time: this cannot be reversed. Moreover, in the liquid state and in water [4], self-organization leads to ordered macrostates poor in microstates.

\section{CONCLUDING REMARKS}

Ageno overcomes the point of view of Boltzmann, which is only related to perfect spheres, not contained inside walls.

We agree with Ageno only for the gas state. In liquids, and namely in water, or in living systems, self-organization plays a completely different fundamental role: in this case irreversibility is related to the origin of life and the capability of dissipative living structures to self-organize.

Irreversible processes may lead to a new dynamic state of matter that Prigogine called dissipative structures: 'They manifest a coherent supermolecular character which leads to new, quite spectacular manifestations.' Anyway dissipative structures cannot be created in isolated systems (no exchange of matter and energy) where entropy can only increase. Whereby in both closed systems (exchange of energy but not matter) and open systems (exchange of matter and energy), dissipative structures can be created and entropy may decrease, provided that a flux of energy of any type is present, and that the system is capable of organizing itself, as does water, with its own intermolecular structure (e.g. hydrogen bonds), giving rise to dissipative structures at the supermolecular level. Prigogine underlines that to extend thermodynamics to non-equilibrium processes, we need an explicit expression for entropy production (see eqn (1)). Once this assumption is accepted we obtain for P, the entropy production per unit time:

$$
P=d_{i} S / d t=\sum_{\rho} J_{\rho} X_{\rho} \geq 0
$$

where $J_{\rho}$ are the rates of the various irreversible processes involved (chemical reactions, heat flow, diffusion) and $X_{\rho}$ are the corresponding generalized forces (affinities, temperature gradients, chemical potential gradients). This is Prigogine's basic formula for the macroscopic thermodynamics of irreversible processes. 
In conclusion, we may say that in water, in liquids and in living systems, containing at least $70 \%$ of water, the most probable state is an ordered one arising from chaos via self-organization. This probability is based on previous events and takes into account the history of the system under study [5].

\section{REFERENCES}

[1] Georgescu-Roegen, N., Energy and Economic Myths, Pergamon Press: New York, 1976.

[2] Prigogine, I. \& Stengers, I., La Nouvelle Alliance. Métamorphose de la Science, Gallimard: Paris, 1979.

[3] Ageno, M., Le Origini Della Irreversibilità, Bollati Boringhieri: Torino, 1992.

[4] Magnani, A., Marchettini, N., Ristori, S., Rossi, C., Rossi, F., Rustici, M., Spalla, O. \& Tiezzi, E., Chemical waves and pattern formation in the 1,2-dipalmitoyl-sn-glycero-3-phosphocholine/ water lamellar system. J. Am. Chem. Soc., 126, pp. 11406-11407, 2004.

[5] Tiezzi, E., Steps Towards an EvolutionaryPhysics, WIT Press: Southampton, 2006. 